\title{
Relación entre la prueba de agilidad específica en taekwondo (tsat), la fuerza explosiva y la velocidad líneal en 5-m atletas de taekwondo de ambos sexos Relationship between the specific agility test in taekwondo (tsat), explosive strength and 5-m linear speed in taekwondo athletes of both sexes \\ *Alex Patricio Ojeda-Aravena, ${ }^{*}$ Jairo Azócar-Gallardo, ${ }^{* * *}$ Claudio Hérnandez-Mosqueira, ${ }^{* *}$ Tomás Herrera-Valenzuela *Universidad de Los Lagos (Chile), **Universidad Santo Tomás (Chile), ***Universidad Adventista (Chile)
}

Resumen. El taekwondo (TKD) es caracterizado como un deporte intermitente y de alta-intensidad donde predominan la ejecución de movimientos rápidos y explosivos, con desplazamientos continuos y cambios de dirección (COD). Objetivo. El propósito de este estudio fue examinar la asociación entre la prueba TSAT y la fuerza explosiva y la velocidad lineal en 5-M en competidores de TKD de nivel nacional de ambos sexos. Material y métodos. A través de un diseño correlacional 14 competidores de ambos sexos que compiten habitualmente en torneos de nivel regional y nacional, (edad $19 \pm 3$ años; talla $164 \pm 10 \mathrm{~cm}$; peso corporal $62 \pm 10 \mathrm{~kg}$; experiencia $7.2 \pm$ 2.9 años) participaron en este estudio. Como indicadores de fuerza explosiva se evaluó squat jump (SJ) y countermovement jump (CMJ), además de la velocidad lineal en 5-m (5-M) y la prueba de agilidad específica en taekwondo (TSAT) Resultados. Entre los principales hallazgos de este estudio se documentó una relación directa $(r=.70)$ entre 5-M y TSAT; una relación inversa $(r=-.63)$ entre $\mathrm{SJ}$; CMJ $(r=-.53)$ y TSAT respectivamente. Conclusiones. Nuestros datos muestran que la prueba de TSAT se relaciona con la velocidad lineal en 5-m y la fuerza explosiva. Los entrenadores deberían considerar el entrenamiento de estas cualidades, utilizando ejercicios similares para desarrollar la agilidad en competidores de TKD.

Palabras Claves. Deportes de combate, cambio de dirección, rendimiento físico, fuerza.

\begin{abstract}
Taekwondo (TKD) is characterized as an intermittent and high-intensity sport in which predominates the execution of fast and explosive movements, with continuous movements and changes of direction (COD). Objective. The purpose of this study was to examine the association between the TSAT test, explosive strength, and 5-M line speed in national level TKD competitors of both sexes. Material and method. Through a correlational design, 14 competitors of both sexes who regularly compete in regional and national tournaments (age $19 \pm 3$ years; size $164 \pm 10 \mathrm{~cm}$; body weight $62 \pm 10 \mathrm{~kg}$; experience $7.2 \pm 2.9$ years), participated in this study. As indicators of explosive strength, squat jump (SJ) and countermovement jump (CMJ) were evaluated, as well as the 5-m linear speed (5M) and the taekwondo-specific agility test (TSAT). Results. Among the main findings of this study, a direct relationship $(r=.70)$ between 5-M and TSAT and an inverse relationship $(r=-.63)$ between SJ, CMJ $(r=-.53)$, and TSAT were documented, respectively. Conclusions. Our data shows that the TSAT test is related to $5-\mathrm{m}$ linear velocity and explosive force. Coaches should consider training these qualities, using similar exercises to develop agility in TKD competitors.
\end{abstract}

Keywords. Combat sports, change of direction, physical performance, strength.

\section{Introducción}

El taekwondo (TKD) olímpico es descrito como un deporte de combate complejo de carácter intermitente y de altaintensidad (relación esfuerzo : pausa; 1:7 s a 1:2) (Santos et al., 2011; da Silva Santos \& Franchini, 2018; Matsushigue et al., 2009), físicamente demandante y que requiere gran habilidad atlética en los atletas (Rocha et al., 2017). Por ejemplo, durante el combate, los atletas realizan acciones motoras de alta-intensidad y de de corta duración (1 a 3 s) durante el ataque (Bridge et al., 2014) seguido de periodos de no-ataque (preparación del ataque o pausas) (Franchini et al., 2019; Santos et al., 2011). Entre las técnicas más utilizadas destaca la patada semicircular o bandal-tchagi (Moreira et al., 2016; Matsushigue et al., 2009; da Silva Santos \& Franchini 2018; (Castro-Garrido et al. 2020) por lo que los atletas requieren de una técnica adecuada, balance, tiempo de reacción para anticipar al oponente (Rocha et al., 2017). A su vez, y dado que la finalidad de este deporte es intentar golpear o patear el torso y la cabeza del oponente y conseguir la mayor cantidad de puntos o el knockout técnico (Tornello et al., 2014) necesitan de una fuerza mínima para ser considerados como puntos (Vargas 2013) y/o generar una adecuada potencia muscular para lograr el knockout y la optima ejecución de

Fecha recepción: 14-03-20. Fecha de aceptación: 08-05-20 Alex Patricio Ojeda-Aravena alex.ojeda@ulagos.cl los movimientos (Rocha et al., 2017) (Moreira, Goethel, y Gonçalves 2016). Además, aceleran y desaceleran continuamente de forma multidireccional de forma repentina (Taskin \& Akkoyunlu, 2016) desplazando el peso corporal hacia delante, hacia atrás y de forma lateral para atacar y defender durante el combate (Chaabene et al., 2018) por lo que la agilidad y la aceleración también han sido sugeridas como habilidadees relevantes en este deporte (Rocha et al., 2017). En este contexto, es de interés para los entrenadores y científicos del deporte identificar la relación entre las habilidades físicas determinantes en este deporte, con la finalidad de desarrollar los atributos físicos específicos para el éxito competitivo en este deporte (Bridge et al., 2014; Chaabene et al., 2018; Vargas y Jiménez 2020).

De acuerdo a lo anterior, la agilidad en particular, es una habilidad compleja, que puede ser definida como un movimiento rápido de todo el cuerpo con cambio de velocidad o dirección en respuesta a un estímulo (Asadi et al., 2016) y que integra las demandas físicas (fuerza y acondicionamiento), procesos cognitivos (aprendizaje motor) y habilidades técnicas (biomecánica) (Asadi et al., 2016). De la agilidad deriva la habilidad de cambio de dirección (COD), que se refiere a un movimiento donde no se requiere una reacción inmediata a un estímulo, por lo que el COD se planifica previamente (Asadi et al., 2016). Entre las demandas físicas, está aceptado en estudios anteriores que esta habilidad se ve influenciada por la fuerza, la potencia y la velocidad (Sheppard 
\& Young 2006). En este sentido, se ha observado una relación grande entre la velocidad de sprint y las pruebas de COD de forma consistente en la bibliografía en deportes colectivos principalmente (Young \& Farrow, 2006). Mientras que su relación con la fuerza explosiva y la potencia muscular es aún controversial (Sheppard \& Young, 2006). La evidencia previa reporta diferentes pruebas de COD entre las que destacan el T-test, Illinois, Zig-Zag, 5-0-5 entre otras, en las que su validez se fundamenta de manera ecológica según las características de los deportes (Nimphius et al., 2018). De manera específica en TKD recientemente se diseñó la prueba específica de agilidad en taekwondo (TSAT) con la finalidad de evaluar esta habilidad en particular, verificandose su validez, confiabilidad y sensibilidad (Chaabene et al., 2018). Además, los autores documentaron una relación inversa moderada con la prueba de saltabilidad countermovement jump (CMJ), y alta con la prueba squat jump (SJ), y una relación directa grande con la prueba velocidad lineal en 5-M (5-M) entre otras variables analizadas. Sin embargo, entre las limitaciones del estudio los autores señalan que la muestra incluyó solo atletas de élite, por lo que sugieren realizar más estudios para verificar la relación con las cualidades físicas mencionadas en diferentes niveles y edades.

En consencuencia, hipotetizamos que la prueba TSAT se relacionará con la velocidad lineal, SJ y con CMJ. Por tanto, el objetivo del presente estudio fue examinar la asociación entre la prueba TSAT, la fuerza explosiva y la velocidad lineal en 5-M en competidores de TKD de nivel nacional de ambos sexos. Los resultados esperados podrían ser relevantes porque proporcionarían orientación a los antrenadores sobre la relación entre las habilidades físicas determinantes en este deporte para desarrollarlas de manera óptima.

\section{Material y métodos}

\section{Participantes}

Este fue un estudio transversal de tipo correlacional con selección de la muestra de manera no probabilística por conveniencia diseñado para examinar la relación entre la prueba específica de agilidad en taekwondo (TSAT) y las cualidades de la fuerza explosiva y la velocidad lineal en 5-m en competidores de TKD de nivel nacional de ambos sexos. Los participantes del estudio fueron reclutados al finalizar la pre-temporada de la periodización anual del 2019. Las sesiones de las mediciones se realizaron durante dos días consecutivos. El primer día se evaluaron la talla $(\mathrm{cm})$, los años de experiencia y el peso corporal $(\mathrm{cm})(\mathrm{PC})$. Posterior a 24 horas se realizó la sesión de evaluación de las pruebas squat jump (cm) (SJ), countermovement jump (cm) (CMJ), velocidad lineal en 5-M ( $\left.\mathrm{m} \mathrm{s}^{-1}\right)(5-\mathrm{M})$ y la prueba de agilidad específica en taekwondo (s) (TSAT). Para esto fueron seleccionados 14 competidores de ambos sexos edad $19 \pm 3$ años; talla 164 $\pm 10 \mathrm{~cm}$; peso corporal $62 \pm 10 \mathrm{~kg}$; años de experiencia $7 \pm 3$ años) pertenecientes a una academia de taekwondo de la Región de Los Lagos, Chile, quienes participaron voluntariamente en este estudio. Para ser incluidos debieron cumplir con los siguientes criterios de inclusión: i) entrenamiento sistemático por más de dos años, durante al menos tres veces por semanas, ii) entrenamiento ininterrumpido previo a su inclusión en el estudio durante e» 6 meses, iii) ausencia de lesiones músculo-esqueléticas en los últimos 6 meses, iv) encontrarse preparando competiciones o torneos organizados por la Federación Nacional de Taekwondo deportivo (FEDANAT, Chile), una organización reconocida por la World Taekwondo. Posteriormente para ser incluidos en el análisis posterior se incluyeron solo a los participantes que completaron con éxito las mediciones antropométricas y las mediciones de las variables de rendimiento físico. Todos los participantes fueron informados acerca de los riegos y beneficios del estudio firmando un consentimiento informado antes de comenzar las mediciones y el estudio. El estudio se condujo de acuerdo a la declaración de Helsinki.

\section{Instrumentos}

Mediciones antropométricas. La talla $(\mathrm{cm})$ se evaluó a través de un estadiómetro (Bodymeter 206) con precisión de $1 \mathrm{~mm}$ siguiendo los protocolos estándares. Brevemente los participantes se ubicaron sin zapatos, con los talones unidos, espalda y glúteos tocando la superficie vertical del estadiómetro y la cabeza colocada en el plano de Frankfort. Por otro lado, el peso corporal ( $\mathrm{kg}$ ) se evaluó mediante escala eléctrica de biompedancia (InBody120, tetrapolar 8-point tactile electrodes system, model BPM040S12F07, Biospace, Inc., USA, to $0.1 \mathrm{~kg})$.

Squat jump (SJ). Se utilizó SJ para evaluar la fuerza explosiva concéntrica del tren inferior a través de la altura máxima alcanzada $(\mathrm{cm})$ del salto vertical. La prueba SJ se realizó utilizando una plataforma de contacto electrónica (Ergojump; Globus, Codogne, Italy) con una precisión de 0.01 m. Durante el SJ se les indicó a los participantes que apoyaran las manos sobre las caderas, los pies bien separados, adoptaran una posición de rodilla flexionada (aproximadamente 90 grados) durante 3 segundos, y luego realizaran un salto vertical de esfuerzo máximo. Todos los participantes recibieron instrucciones de aterrizar en posición vertical y flexionar las rodillas después del aterrizaje (Ramírez-Campillo et al., 2013). Se completaron tres intentos, con un minuto de recuperación entre intentos (Moran et al., 2017) y el intento de mejor rendimiento se utilizó para el análisis estadístico posterior.

Countermovement jump(CMJ). Se utilizó CMJ para evaluar el ciclo de acortamiento-estiramiento lento utilizando la altura máxima alcanzada $(\mathrm{cm})$ del salto vertical. La prueba CMJ se realizó utilizando una plataforma de contacto electrónica (Ergojump; Globus, Codogne, Italy) con una precisión de $0.01 \mathrm{~m}$. Durante el CMJ se les indicó a los participantes que apoyaran las manos sobre las caderas, los pies bien separados; los participantes realizaron un movimiento hacia abajo (no se impuso ninguna restricción sobre el ángulo de rodilla alcanzado) seguido de un salto vertical de esfuerzo máximo. Todos los sujetos recibieron instrucciones de aterrizar en posición vertical y flexionar las rodillas después del aterrizaje (Ramírez-Campillo et al., 2013). Se completaron tres intentos, con un minuto de recuperación entre intentos (Moran et al., 2017) y el intento de mejor rendimiento se utilizó para el análisis estadístico posterior.

Velocidad lineal en 5-m (5-M). Para evaluar la velocidad lineal se registraron los tiempos $\left(\mathrm{m} \mathrm{s}^{-1}\right)$ en $0-5 \mathrm{M}$ (Cronin et al., 2007). Las pruebas se realizaron en una superficie de gimnasio de madera. Los participantes adoptaron una posición de salida similar a la de una carrera de velocidad y co- 
menzaron a correr en forma voluntaria. Se registró la velocidad mediante sistema de cronometraje automático mediante fotocélulas electrónicas (Brower Timing System, Salt Lake City, UT). con precisión de $0.001 \mathrm{~s}$. Las puertas (gates) fueron posicionadas a 1-m de distancia sobre el suelo. Los competidores completaron dos intentos de práctica después del calentamiento general, para familiarizarse con el dispositivo de medición. Luego, completaron tres ensayos máximos, con seis minutos de recuperación entre ensayos (Brady et al., 2019) utilizando el mejor para análisis estadístico.

Agilidad específica en Taekwondo (TSAT). El COD se evaluó mediante la prueba de agilidad específica en Taekwondo (TSAT) siguiendo recomendaciones previas (Chaabene et al., 2018). Desde una posición de guardia con ambos pies detrás de la línea de inicio / final, el participante tuvo que: 1) moverse hacia adelante en la posición de guardia sin cruzar los pies lo más rápido posible hacia el punto central, 2) girar hacia el compañero 1 adoptando un desplazamiento lateral y realizar una patada circular con la pierna izquierda (es decir, patada semicircular; dollyo-chagi); 3) moverse hacia el compañero 2 y realizar una patada semicircular con la pierna derecha (es decir, patada delantera; dollyo-chagi); 4) Regresar al centro; 5) moverse hacia adelante en la posición de guardia y realizar una patada doble (es decir, narae-chagi) hacia la pareja 3, y 6 ) retroceder a la línea de inicio / finalización en posición de guardia. Los compañeros de combate 1 y 2 tuvieron un foco de patada, mientras que el compañero 3 tuvo dos focos de patada. Los compañeros de combate fueron instruidos para mantener el foco de pateo a la altura del torso del participante evaluado. Si el participante no siguió estas instrucciones (por ejemplo, cruzó un pie delante del otro durante los diversos desplazamientos o no pudo tocar el foco de patada con fuerza al patear), la prueba finalizó y reinició después de 3 minutos de período de recuperación. El tiempo necesario para completar la prueba en segundos (s) se registró mediante un sistema de temporización electrónico (Brower Timing Systems, Salt Lake City, EE. UU.) colocado de forma paralela en la línea de inicio/ final de la prueba. Se completaron tres ensayos para cada participante y se utilizó el mejor para su análisis.

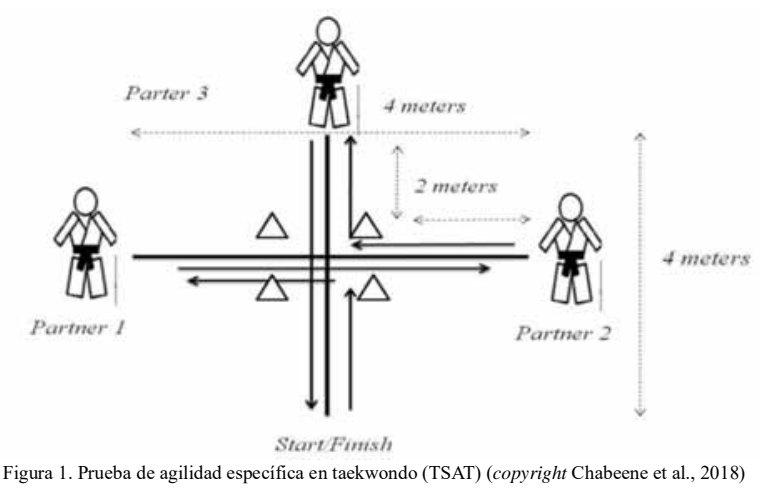

\section{Procedimiento}

Las mediciones se realizaron posterior a 48 horas del último entrenamiento físico durante la última semana del mes de marzo de 2019. Dos semanas previas los participantes completaron una sesión de familiarización con los protocolos de medición del rendimiento físico para reducir el efecto de aprendizaje de los procedimientos. Todas las pruebas estandarizadas fueron programadas entre las 9:00 y las 11:00 horas de la mañana durante el sábado 29 y el Domingo 30 de marzo de 2019 en un recinto cerrado y realizadas por un profesional calificado quien estuvo cegado de los participantes del estudio al momento de las mediciones. Entre las condiciones de vestimenta se solicitó a los participantes utilizar camiseta, pantalón corto y calzado para correr. Previo a las mediciones todos los participantes fueron instruidos para que (a) descansaran de manera adecuada la noche anterior durmiendo 8 o más horas, (b) no consumieran bebidas estimulantes previa las mediciones, (c) consumieran $\sim 2$ litros de agua durante el día anterior, (d) alimentarse de forma habitual sin realizar modificaciones en la dieta.

Durante el día de las mediciones los participantes fueron instruidos para dar su máximo esfuerzo durante las pruebas. El orden de las pruebas fue establecido de acuerdo con la intensidad de cada prueba en el siguiente orden: SJ, CMJ, 5M, y TSAT. Un calentamiento típico en este deporte fue realizado con $\sim 15$ minutos de duración que consistió en movilidad articular, trote suave por 5 minutos, estiramientos dinámicos y patadas de baja intensidad. El mejor de 3 intentos fue grabado para todas las mediciones exceptuando TSAT donde se grabó el resultado único obtenido en cada prueba. Un intervalo de descanso de 5 a 10 minutos fue aplicado entre cada prueba para reducir los efectos de fatiga (RamírezCampillo et al., 2013).

\section{Análisis estadístico de los datos}

Todos los datos descriptivos y del rendimiento físico de los competidores analizados fueron presentados utilizando la media \pm desviación estándar. La normalidad de los datos fue verificada utilizando la prueba de Shapiro-Wilk. Posteriormente un T-test no pareado fue aplicado para establecer las diferencias entre las variables descriptivas del estudio entre hombres y mujeres. Para examinar la relación entre la prueba TSAT y las variables SJ; CMJ y 5-M se utilizó la correlación de Pearson $(r)$. De 0 a .30, o 0 a «.30 se consideró [baja]; .31 a .49, o «.31 a «.49 [moderada]; .50 a .69, o «.50 a «.69 [grande]; 0.70 a $0.89, \mathrm{o} \ll .70$ a «.89 [muy grande]; $\mathrm{y} .90 \mathrm{a}$ $1.0, \mathrm{o} \ll .90$ a 1.0 una correlación [casi perfecta a perfecta] (Hopkins 2002) y fueron expresados con el $90 \%$ del coeficiente de intervalo $(\mathrm{CI})$. Además se estimó el coeficiente de determinación $\left(\mathrm{R}^{2}\right)$ para expresar el porcentaje de cambio en la prueba TSAT según el cambio en las variables de SJ; CMJ y 5-M (Borda Pérez et al., 2013). Todos los análisis estadísticos se realizaron utilizando el paquete estadístico GraphPad Prism (versión 6.0, San Diego, California). El nivel de significancia estadística se estableció en $\mathrm{p}<.05$.

\section{Resultados}

La tabla 1 muestra las variables descriptivas del estudio. En promedio los atletas hombres tenían una edad de $20.8 \pm 2.5$ años, una talla de $172.1 \pm 8.8 \mathrm{~cm}$, un peso corporal de $68.8 \pm 9.3 \mathrm{~kg}$ y una experiencia de $7.4 \pm 2.7$ años. En tanto las mujeres tenian una edad de $17.7 \pm 2.8$ años, una talla de $157.6 \pm 6.0 \mathrm{~cm}$, un peso corporal de $56.9 \pm 8.0 \mathrm{~kg} \mathrm{y}$ una experiencia de $7.1 \pm 3.2$ años. A su vez, no se observaron diferencias significativas entre la edad (años) de hombres y mujeres ( $p>51$ ). Tampoco no se observaron dife- 
rencias significativas entre los años de experiencia de hombres y mujeres ( $p>.86$ ). La tabla 2 muestra las variables de rendimiento del estudio. En términos generales los atletas de ambos sexos ejecutaron la prueba TSAT en 7.82 \pm 0.86 segundos, la prueba de velocidad lineal en 5 -m en $1.20 \pm 0.07 \mathrm{~m} \mathrm{~s}-1$, saltaron en SJ $28.6 \pm 6.6 \mathrm{~cm}$ y en CMJ $32.5 \pm 6.3 \mathrm{~cm}$.

La tabla 3 muestra las correlaciones entre las variables 5-M ; SJ y CMJ con la variable TSAT. Una relación grande significativa ( $r=.70 ; p<0.001 ; R^{2}=.49 ; C l 90 \% .09$ a .92) se observó entre la velocidad lineal en 5-m y la prueba TSAT. A su vez, una relación inversa grande significativa se documentó entre la prueba de salto $\mathrm{S})(\mathrm{r}=-.63 ; \mathrm{p}<.01 ; \mathrm{Cl}$ $90 \% 0.2$ a $-.90 ; R^{2}=.40$ ) y la prueba TSAT. Finalmente se observó una relación inversa grande significativa entre el rendimiento de la prueba $C M$ J y la agilidad específica ( $r=$ 56; $p<.03 ; \mathrm{Cl} 90 \% .12$ a $-88 ; \mathrm{R}^{2}=.32$ )

La figura 1 muestra en detalle las correlaciones entre las variables $5 \mathrm{M}$; SJ y CMJ con la variable TSAT.

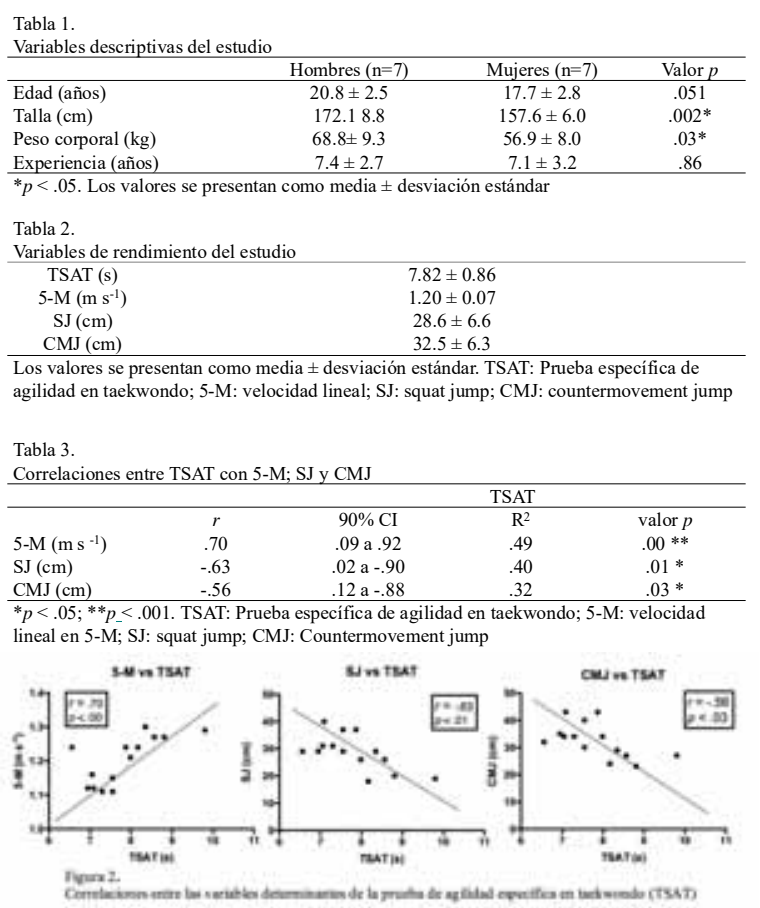

\section{Discusión}

El propósito de este estudio fue examinar la asociación entre la prueba TSAT, la fuerza explosiva y la velocidad lineal en 5-M en competidores de TKD de nivel nacional de ambos sexos. Entre los principales hallazgos se documentó una asociación muy grande $(p<.001)$ entre la prueba 5-M y la prueba TSAT. A su vez, una asociación inversa grande $(p<.01)$ entre SJ y TSAT y una asociación inversa grande $(p<.03)$ entre CMJ y TSAT. Estos resultados sugieren que la prueba de TSAT podría estar influenciada por la velocidad lineal en 5-M y por la fuerza explosiva concéntrica más que el ciclo acortamiento-estiramiento. Por lo que los entrenadores deberían considerar el entrenamiento de estas tres cualidades, utilizando ejercicios similares para desarrollar la agilidad en los competidores de TKD. Sin embargo, se sugiere hacer énfasis en el entrenamiento concéntrico.
En relación a la prueba de 5-M se observó una correlación directa con la prueba TSAT donde el $49 \%$ del cambio en el tiempo de ejecución de la prueba de 5-M podría haber influenciado el cambio en los tiempos de ejecución de la prueba TSAT. Estos datos difieren con los observado por Chaabene et al. (2018) quienes documentaron en atletas élite un rendimiento superior en la prueba de $5-\mathrm{M} 1.0 \pm 0.1 \mathrm{~s}$. Sin embargo, a pesar de la diferencia en los tiempos de ejecución de 5-M, los datos de la correlación observada en este estudio, concuerdan con los autores previamente señalados. Quienes observaron una relación grande $(r=.52)$ con 5-M (Chaabene et al., 2018). Estos datos complementan la relación grande $(r<.71)$ entre la velocidad de sprint y las pruebas de COD consistentemente observada en estudios previos (Young \& Farrow, 2006). La velocidad y la habilidad de acelerar de manera lineal es una habilidad subyacente en la agilidad en los deportes colectivos (Dawes 2019) y también de acuerdo a nuestros resultados implicada en el TKD.

Por otro lado, con respecto al SJ se reportó una relación inversa grande con la prueba TSAT. Un $40 \%$ de los cambios observados en SJ podrían explicar los cambios en el rendimiento TSAT. Estos datos concuerdan con lo reportado por Chaabene et al. (2018) quienes documentaron valores similares en la altura de salto alcanzada en SJ y una correlación $\operatorname{similar}(r=$-.62) (Chaabene et al., 2018). El desempeño en la prueba SJ refleja las características concéntricas de la fuerza explosiva que intervienen durante la prueba TSAT. Esta característica se ve reflejada en el empuje horizontal donde interviene una triple extensión de cadera, rodilla y tobillo para vencer la fuerza de gravedad e impulsarse hacia delante durante la prueba TSAT (Dawes 2019). Además, la manifestación concéntrica se observa en la activación del bíceps femoral, semitendinoso de manera concéntrica al momento del impacto de la patada bandal-tchagi, utilizada en esta prueba, para desacelerar y estabilizar la rodilla (Valdes-Badilla et al., 2018).

En cuanto a la prueba $\mathrm{CMJ}$ se documentó una relación grande inversa con la prueba TSAT. En tanto, un 32\% de los cambios obtenidos en esta prueba podrían explicar el rendimiento obtenido en TSAT. Estos valores son similares a los reportados por (Chaabene et al., 2018) $(r=-.43)$ aunque superiores entre CMJ y TSAT. La fuerza explosiva es un prerrequisito para la ejecución explosiva de las patadas (Chaabene et al., 2018). Además, se sugiere que la utilización del ciclo acortamiento-estiramiento estaría presente en las desaceleraciones y aceleraciones constantes al desplazar y/ o patear durante el combate, requeriendo más estudios al respecto.

Este es el primer estudio de acuerdo a nuestro conocimiento en analizar la relación entre algunas de las cualidades físicas y la prueba específica TSAT en competidores de TKD chilenos de nivel nacional de ambos sexos.

No obstante, el presente estudio no estuvo falto de limitaciones. Al ser un estudio correlacional no pudo determinarse que la respuesta en la velocidad lineal en 5-M y la fuerza explosiva sean las causas de la respuesta en los resultados obtenidos en TSAT. Además, se utilizó una muestra reducida y por conveniencia por lo que no se pueden generalizar los resultados obtenidos ya que, al ser una muestra reducida, los resultados presentarían una baja potencia es- 
tadística, lo cual nos lleva a tomar con precaución los resultados. En futuras investigaciones se sugiere, como lo plantean otros autores, utilizar un número mayor atletas de taekwondo de nivel amateur y de élite evaluando otros componentes como variables fisiológicas, nutricionales, de entrenamiento y rendimiento a fin de mejorar la caracterización de este tipo de deportistas.

\section{Conclusiones}

Nuestros datos sugieren que la prueba de TSAT se vería influenciada por la velocidad lineal en 5-m y por la fuerza explosiva concéntrica más que el ciclo acortamiento-estiramiento.

\section{Aplicaciones prácticas}

Entre las aplicaciones prácticas se sugiere que los entrenadores deberían considerar el entrenamiento de estas cualidades, utilizando estrategías como el entrenamiento de fuerza de acuerdo con (Castro-Garrido et al. 2020; da Silva Santos et al. 2020) incluyendo ejercicios balísticos como sentadilla y press de banco, el uso de bandas elásticas. Además del trabajo pliométrico utilizando saltos verticales y horizontales bilaterales y unilaterales haciendo énfasis en los aspectos concéntricos para desarrollar la fuerza explosiva y contribuir a la agilidad en competidores de TKD.

\section{Referencias}

Asadi, A., Arazi, H., Young, W. B., \& de Villarreal, E. S. (2016). The effects of plyometric training on change-of-direction ability: A meta-analysis. International journal of sports physiology and performance, 11(5), 563-573.

Borda Pérez, M., Tuesca Molina, R., \& Navarro Lechuga, E. (2013). Métodos cuantitativos. Herramientas para la investigación en salud 4ed. Universidad del Norte.

Brady, C. J., Harrison, A. J., Flanagan, E. P., Haff, G. G., \& Comyns, T. M. (2019). The Relationship Between Isometric Strength and Sprint Acceleration in Sprinters. International journal of sports physiology and performance, 1(aop), 1-8.

Bridge, C. A., da Silva Santos, J. F., Chaabene, H., Pieter, W., \& Franchini, E. (2014). Physical and physiological profiles of taekwondo athletes. Sports Medicine, 44(6), 713-733.

Castro-Garrido, N., Valderas-Maldonado, C., Herrera-Valenzuela, T., Da Silva, J. F., Guzmán-Muñoz, E., Vásquez-Gómez, J., ... \& López-Fuenzalida, A. (2020). Effects of post-activation potentiation exercises on kicking frequency, fatigue rate and jump performance in taekwondo athletes: a case study (Efectos de los ejercicios de potenciación post activación sobre la frecuencia de pateo, tasa de fatiga y saltabi. Retos, 38(38), 679683.

Chaabene, H., Negra, Y., Capranica, L., Bouguezzi, R., Hachana, Y., Rouahi, M. A., \& Mkaouer, B. (2018). Validity and reliability of a new test of planned agility in elite taekwondo athletes. The Journal of Strength \& Conditioning Research, 32(9), 2542-2547.

Cronin, J. B., Green, J. P., Levin, G. T., Brughelli, M. E., \& Frost, D. M. (2007). Effect of starting stance on initial sprint performance. Journal of Strength and Conditioning Research, 21(3), 990.

da Silva Santos, J. F., Wilson, V. D., Herrera-Valenzuela, T., \& Machado, F. S. M. (2020). Time-Motion Analysis and
Physiological Responses to Taekwondo Combat in Juvenile and Adult Athletes: A Systematic Review. Strength \& Conditioning Journal, 42(2), 103-121.

Dawes, J. (Ed.). (2019). Developing agility and quickness. Human Kinetics Publishers.

Franchini, E., Cormack, S., \& Takito, M. Y. (2019). Effects of High-Intensity Interval Training on Olympic Combat Sports Athletes' Performance and Physiological Adaptation: A Systematic Review. The Journal of Strength \& Conditioning Research, 33(1), 242-252.

Hopkins, W. G. (2002). A new view of statistics: Effect magnitudes. Retrieved February, 14, 2005.

Matsushigue, K. A., Hartmann, K., \& Franchini, E. (2009). Taekwondo: Physiological responses and match analysis. The Journal of Strength \& Conditioning Research, 23(4), 11121117.

Moran, J., Sandercock, G. R., Ramírez-Campillo, R., Todd, O., Collison, J., \& Parry, D. A. (2017). Maturation-related effect of low-dose plyometric training on performance in youth hockey players. Pediatric Exercise Science, 29(2), 194-202.

Nimphius, S., Callaghan, S. J., Bezodis, N. E., \& Lockie, R. G. (2018). Change of direction and agility tests: Challenging our current measures of performance. Strength \& Conditioning Journal, 40(1), 26-38.

Ramírez-Campillo, R., Andrade, D. C., \& Izquierdo, M. (2013). Effects of plyometric training volume and training surface on explosive strength. The Journal of Strength \& Conditioning Research, 27(10), 2714-2722.

Rocha, F., Costa, A., Louro, H., \& Conceição, A. (2017). The relationship between bandal chagui power with mechanical efficiency in taekwondo athletes. Sci. Int.(Lahore), 29(6), 12311236.

Santos, J. F. D. S., \& Franchini, E. (2018). Frequency Speed of Kick Test Performance Comparison Between Female Taekwondo Athletes of Different Competitive Levels. Journal of strength and conditioning research, 32(10), 2934-2938.

Santos, V. G., Franchini, E., \& Lima-Silva, A. E. (2011). Relationship between attack and skipping in taekwondo contests. The Journal of Strength \& Conditioning Research, 25(6), 17431751.

Sheppard, J. M., \& Young, W. B. (2006). Agility literature review: Classifications, training and testing. Journal of sports sciences, 24(9), 919-932.

Taskin, M., \& Akkoyunlu, Y. (2016). Effect of anaerobic power on quickness in women national taekwondo athletes. Ovidius University Annals, Series Physical Education and Sport/Science, Movement and Health, 16(2 SI), 701-706.

Tornello, F., Capranica, L., Minganti, C., Chiodo, S., Condello, G., \& Tessitore, A. (2014). Technical-tactical analysis of youth Olympic Taekwondo combat. The Journal of Strength \& Conditioning Research, 28(4), 1151-1157.

Valdes-Badilla, P., Barramuno Medina, M., Astudillo Pinilla, R., Herrera-Valenzuela, T., Guzman-Munoz, E., Perez-Gutierrez, M., ... \& Martinez Salazar, C. (2018). Differences in the electromyography activity of a roundhouse kick between novice and advanced taekwondo athletes. Ido Movement for Culture. Journal of Martial Arts Anthropology, 18(1), 31-38.

Vargas, P. C. (2013). Respuestas y adaptaciones fisiológicas en el entrenamiento de taekwondo. Una revisión sistemática. Pensar en movimiento: Revista de Ciencias del Ejercicio y la Salud, 11(2), 1-19.

Vargas, P. C., \& Jiménez, J. M. (2020). The association between sleep efficiency and physical performance in taekwondo athletes. Retos, (37), 227-232.

Young, W., \& Farrow, D. (2006). A review of agility: Practical applications for strength and conditioning. Strength and conditioning journal, 28(5), 24. 